DOI: $10.3901 / J M E .2018 .03 .008$

\title{
重型工作面输送机变频驱动与传动"
}

\author{
李 阳 ${ }^{1}$ 何柏岩 ${ }^{1}$ 袁鹏飞 $^{1}$ 聂 锐 ${ }^{1}$ 李国平 $^{2}$ \\ (1. 天津大学机构理论与装备设计教育部重点实验室 天津 300354; \\ 2. 中煤张家口煤矿机械有限责任公司 张家口 075025)
}

\begin{abstract}
摘要: 为解决工作面输送机启动困难、多机驱动功率不均衡等问题, 可采用变频驱动这一软启动方式来改善其启动性能, 但 变频控制策略与整机动力学的适配协同是有效发挥变频驱动优势的重要课题。根据变频电动机的机械特性曲线建立电动机模 型, 将输送机的链传动系统离散成多个 Kelvin-Vogit 模型。利用 Matlab/Simulink 软件平台, 建立了输送机整机系统模型, 分

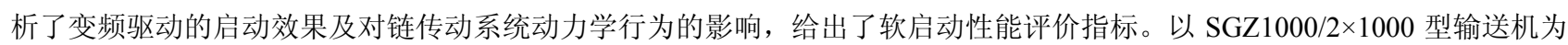
例, 对满载启动工况进行了数值仿真。分析不同控制参数对输送机启动过程的影响, 得到了最优控制策略。与同等规格/工况 下的液力偶合器驱动对比表明: 合理的变频驱动控制策略可以明显改善输送机软启动性能, 启动平稳且减小了系统机械冲击, 并可实现多机驱动功率平衡, 有效解决了重载难启动等问题。
\end{abstract}

关键词: 工作面输送机; 变频驱动; 系统模型; 性能指标

中图分类号: TD528

\section{Variable Frequency Drive and Transmission System of the Heavy Armoured Face Conveyor}

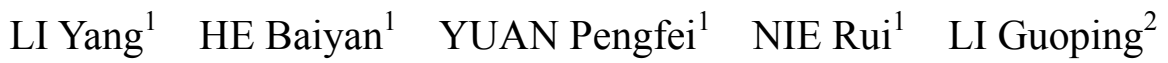 \\ (1. Key Laboratory of Mechanism Theory and Equipment Design of Ministry of \\ Education, Tianjin University, Tianjin 300354; \\ 2. China Coal Zhangjiakou Coal Mining Machinery Co., Ltd., Zhangjiakou 075025)
}

\begin{abstract}
The variable frequency drive has been adopted to solve the problems of smooth starting and imbalance of multi-driving for the armoured face conveyor recently. In order to take full advantage of this soft start method, the obtaining of frequency control strategy and the systematic dynamic analysis should be combined. The electromechanical model of the variable frequency motor is established according to the mechanical characteristic curve and the chain transmission system is discretized into multiply Kelvin-Vogit models. The systematic electromechanical model of an armoured face conveyor is established by Matlab/Simulink. Then, the effect of the variable frequency drive on the dynamic behaviors of the chain transmission system is analyzed, and the performance evaluation index of the soft start is introduced. A numerical simulation about the SGZ1000/2×1000 conveyer on the full loading condition is provided as an example. The influence of control parameters on the starting process is analyzed, and an optimal control strategy is obtained. After compared with the system driven by the motor and hydraulic coupling under the same working condition, it can be concluded that reasonable control parameters can obviously improve the soft start performances, ensure a smooth startup, reduce mechanical impact, achieve the power balance of multi-driving, and solve the startup problems under heavy loads.
\end{abstract}

Key words: armoured face conveyor; variable frequency drive; systematic model; performance evaluation

\section{0 前言}

随着煤炭开采集约化、大型化, 工作面输送机 的装机功率越来越大, 目前最大已达 $3 \times 2000 \mathrm{~kW}$ 。

* 国家自然科学基金资助项目(51375330)。20170612 收到初稿, 20171019 收到修改稿
传统驱动方式已不能满足输送机运行要求, 存在以 下问题: (1) 重载难启动; (2) 强大的机械冲击和电 网冲击; (3) 多机驱动功率不均衡。为改善输送机传 动系统的拖动特性, 变频驱动作为一种理想的软启 动方式已成为研究的热点。

国外学者 DU 等 ${ }^{[1]}$ 从节约能源潜力、经济因素、 潜在障碍等方面论证了变频调速技术在煤矿井下 的应用; JUNGHANS ${ }^{[2]}$ 介绍了变频技术在带式输送 
机上的应用; WAUGE ${ }^{[3]}$ 研究并建立了工作面输送机 驱动系统的仿真模型; DOLIPSKI ${ }^{[4]}$ 对电源电压和频 率的改变对输送机负载均匀性的影响展开了研究。 国内的宋伟刚等 ${ }^{[5-6]}$ 针对长距离带式输送机, 提出了 控制启、停过程的设计方法; NIE 等 ${ }^{[7-8]}$ 对工作面输 送机链传动系统开展了动力学行为研究, 并开发出 相应的软件平台; 车林仙 ${ }^{[9]}$ 对刮板输送机传动设计 问题展开了研究; CHENG 等 ${ }^{[10]}$ 研究并建立了双子 链传动系统的数学模型和动力学分析模型; 孙阳辉 ${ }^{[11]}$ 研究了工作面输送机各部件间参数的匹配问题; 毛君等 ${ }^{[12-13]}$ 针对输送机不同启动方式进行了仿真, 得出合适的启动方式能够大大降低启动动负荷; 李 小凡 ${ }^{[14]}$ 建立了工作面输送机变频电动机电学模型, 并对满载工况进行了数值仿真。

目前对输送机变频驱动的研究存在以下不足: 多数只针对变频技术进行理论性介绍, 缺乏系统性 建模与数据支撑; 只侧重变频电动机的研究而忽略 了链传动系统对整机动力学行为的影响。鉴于此, 本文建立了计及变频电动机及链传动系统的输送机 整机动力学模型, 针对不同控制参数进行满载启动 工况仿真, 从而得到最优控制策略。仿真结果与同 等工况下液力偶合器驱动进行对比, 验证了变频启 动在输送机应用中具有很好的调速性能、高可靠性、 高启动转矩、良好的功率平衡等优势, 从而为大功 率输送机驱动方式的选取及变频电动机控制策略的 研究提供理论依据与工程指导。

\section{1 工作面输送机传动系统}

工作面输送机是综采工作面 “三机” 装备的核 心设备 ${ }^{[15]}$, 起着承载、运输以及为采煤机提供导向 和为液压支架提供推移支撑的作用。

变频驱动的输送机传动系统见图 1, 主要由变 频电动机、减速器、链传动系统组成。相比于常见 的偶合器/限矩器输送机传动系统, 采用变频电动机 直驱减速器连接的配置形式, 不仅省去液力偶合器, 且提高了传递效率。本文将分别建立变频电动机模

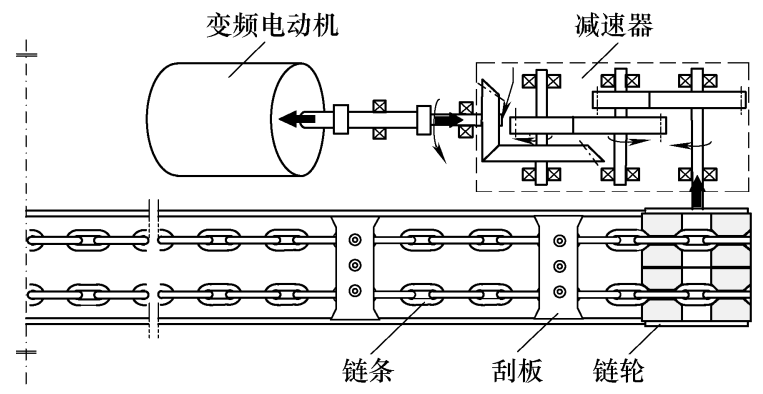

图 1 工作面输送机传动系统简图
型、链传动系统模型, 针对减速器进行简化处理, 忽略功率损失, 并将其转动惯量折算到链轮轴上, 最终建立输送机整机动力学模型, 在此基础上探讨 变频电动机控制策略对整机动力学的影响与优化。

\section{2 变频电动机模型的建立}

根据电动机的电磁特性建立的动力学模型较为 准确, 但其求解时间较长, 电学参数难以准确的测 量, 从而影响模型精度。因此, 针对复杂的混合系 统, 可依据机械特性曲线建立电动机模型, 该模型 仅关注电动机表现出的机械特性, 忽略了其内部复 杂的电磁学关系, 在模型复杂度、求解时间和精度 之间取得了平衡。因此, 本文将根据变频电动机的 机械特性曲线来建立其动力学模型。

\section{1 变频调速原理}

异步电动机转速可表示为

$$
n_{0}=\frac{60 f}{p}
$$

式中, $n_{0}$ 为同步转速; $f$ 为电源频率; $p$ 为电动机 磁极对数。

由上式可知, 电动机转速正比于工作电源频率, 连续改变供电电源的频率, 就可连续平滑地调节电 动机转速。

\section{2 变频电动机机械特性}

工作面输送机是在低速状态下运行的, 但需要 的起动转矩较大, 且输送机基本属于恒转矩负载类 型。基于此, 变频调速采用恒转矩调速, 即维持最 大转矩恒定。图 2 所示为不同电源频率下电动机的 机械特性曲线, 其中 $s$ 为转差率, $s_{\mathrm{m}}$ 为临界转差率, $f_{1} 、 f_{2}$ 为不同电源频率, $T_{\mathrm{st}}$ 为启动转矩, $T_{\mathrm{Mm}}$ 为最大 转矩或临界转矩。

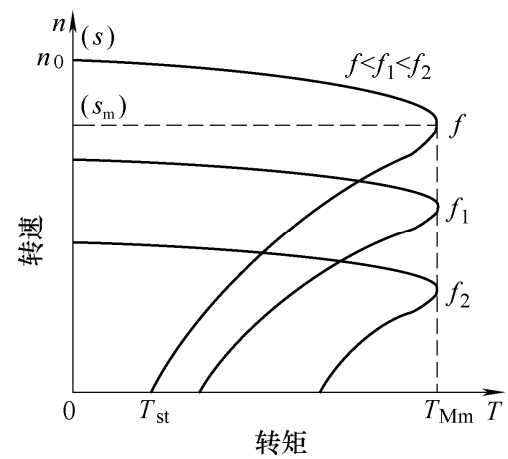

图 2 不同电源频率下电动机的机械特性

分析图 2 可知, 变频电动机的机械特性可认为 是异步电动机的机械特性曲线随频率改变发生平移 而得到。每改变频率，就可得到一条该频率对应的 
机械特性曲线。将一系列曲线组合起来, 便得到变 频电动机的机械特性曲线, 可据此建立变频电动机 的动力学模型。

常采用 KLOSS 公式 ${ }^{[11]}$ 来描述异步电动机的机 械特性。

$$
T_{\mathrm{M}}=2 T_{\mathrm{Mm}} /\left(\frac{s}{s_{\mathrm{m}}}+\frac{s_{\mathrm{m}}}{s}\right)
$$

式中, $T_{\mathrm{M}}$ 为电磁转矩。
由于集肤效应的影响, 该公式用于鼠笼式异步 电动机启动阶段时会产生较大误差。矿井下电动机 多采用鼠笼式异步电动机, 为此, 根据文献[16]对式 (2)进行修正, 从而得到鼠笼式电动机机械特性方程, 以用来描述大功率矿用鼠笼式电动机的机械特性。

由图 2 可以看出, 当电源频率变化时, 电动机 机械特性曲线发生平移, 转差率也随之变化, 由此 可得到变频电动机机械特性方程

$$
\left\{\begin{array}{l}
T_{\mathrm{M}}=\frac{2\left(\frac{T_{\mathrm{Mm}}-T_{\mathrm{Mm} 1} s_{\mathrm{m}}}{1-s_{\mathrm{m}}}+\frac{T_{\mathrm{Mm} 1}-T_{\mathrm{Mm}}}{1-s_{\mathrm{m}}} \cdot s\right)}{s /\left[s_{\mathrm{m}}\left(\frac{1-s_{\mathrm{m} 1}}{1-s_{\mathrm{m}}}+\frac{s_{\mathrm{m} 1}-s_{\mathrm{m}}}{1-s_{\mathrm{m}}} \cdot \frac{s}{s_{\mathrm{m}}}\right)\right]+s_{\mathrm{m}}\left(\frac{1-s_{\mathrm{m} 1}}{1-s_{\mathrm{m}}}+\frac{s_{\mathrm{m} 1}-s_{\mathrm{m}}}{1-s_{\mathrm{m}}} \cdot \frac{s}{s_{\mathrm{m}}}\right) / s} \\
s=1-\frac{n_{\mathrm{e}} p}{60 f}
\end{array}\right.
$$

式中, $T_{\mathrm{Mm} 1}$ 为启动时 $(s=1)$ 的临界转矩; $s_{\mathrm{m} 1}$ 为启动 时 $(s=1)$ 的临界转差率; $n_{\mathrm{e}}$ 为电动机转速。

电动机在负载作用下的运动方程为

$$
T_{\mathrm{M}}-T_{\text {load }}=\frac{\pi I_{\mathrm{M}}}{30} \cdot \frac{\mathrm{d} n_{\mathrm{e}}}{\mathrm{d} t}
$$

式中, $T_{\text {load }}$ 为负载转矩; $I_{\mathrm{M}}$ 为电动机的转动惯量。

由式(1)、(3)、(4)构成变频电动机动力学模型。

\section{3 链传动系统模型的建立}

\section{1 链传动系统的离散化模型}

采用有限段法, 将输送机链传动系统离散成 Kelvin-Vogit 模型, 建立图 3 所示的链传动系统离散 化动力学模型 ${ }^{[7-8,17]}$ 。

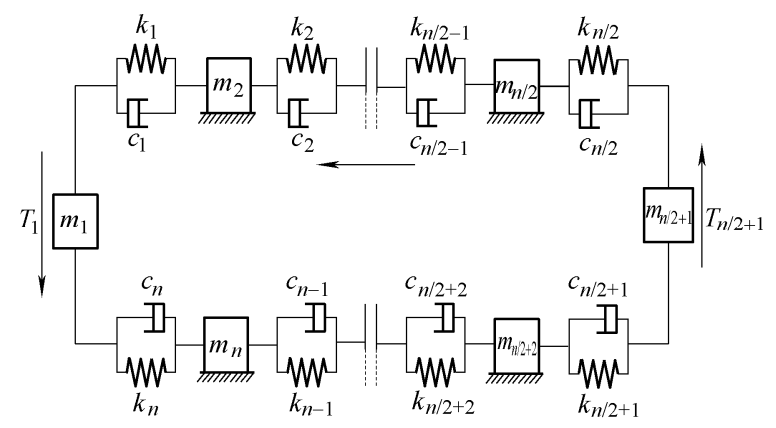

图 3 工作面输送机链传动系统离散化模型

将链传动系统离散成 $n$ 个单元, 其运动方向按图 3 中箭头所示, 选定各单元的运动方向为位移的正方 向。根据牛顿-欧拉方程可得到单元的动力学方程

$$
\begin{gathered}
m_{i} \ddot{x}_{i}-\left[k_{i-1}\left(x_{i-1}-x_{i}\right)+c_{i-1}\left(\dot{x}_{i-1}-\dot{x}_{i}\right)-k_{i}\left(x_{i}-x_{i+1}\right)-\right. \\
\left.c_{i}\left(\dot{x}_{i}-\dot{x}_{i+1}\right)\right]-f_{i}=0 \quad i=2 \cdots n / 2, n / 2+2, \cdots, n
\end{gathered}
$$

式中, $m_{i}$ 为单元 $i$ 的质量; $x_{i}$ 为单元 $i$ 的位移; $\dot{x}_{i}$ 为
单元 $i$ 的速度; $\ddot{x}_{i}$ 为单元 $i$ 的加速度; $k_{i}$ 为单元 $i$ 的 刚度系数; $c_{i}$ 为单元 $i$ 的阻尼系数; $f_{i}$ 为单元 $i$ 中刮 板链条及货载与溜槽和底板的摩擦力。

刚度系数可通过有限元分析得到 ${ }^{[17]}$; 阻尼系数 可通过工程试验获得。

对于头部和尾部的链轮单元, 应用转矩公式得

$$
\begin{gathered}
I_{1} \ddot{\theta}_{1}-\left[k_{n}\left(x_{n}-x_{1}\right)+c_{n}\left(\dot{x}_{n}-\dot{x}_{1}\right)-\right. \\
\left.k_{1}\left(x_{1}-x_{2}\right)-c_{1}\left(\dot{x}_{1}-\dot{x}_{2}\right)\right] \cdot r+T_{1}=0
\end{gathered}
$$

$$
I_{n / 2+1} \ddot{\theta}_{n / 2+1}-\left[k_{n / 2}\left(x_{n / 2}-x_{n / 2+1}\right)+c_{n / 2}\left(\dot{x}_{n / 2}-\dot{x}_{n / 2+1}\right)-k_{n / 2+1}\right.
$$$$
\left.\left(x_{n / 2+1}-x_{n / 2+2}\right)-c_{n / 2+1}\left(\dot{x}_{n / 2+1}-\dot{x}_{n / 2+2}\right)\right] \cdot r+T_{n / 2+1}=0
$$

式中, $I_{1} 、 I_{n / 2+1}$ 分别为头部和尾部链轮的转动惯量; $\ddot{\theta}_{1} 、 \ddot{\theta}_{n / 2+1}$ 分别为头部和尾部链轮的角加速度; $r$ 为 链轮半径; $T_{1} 、 T_{n / 2+1}$ 分别为施加到头部和尾部链轮 上的驱动转矩。

为统一各单元运动方程的形式, 将式(6)、 化成式(5)所示的形式

$$
\begin{gathered}
m_{i} \ddot{x}_{i}-\left[k_{i-1}\left(x_{i-1}-x_{i}\right)+c_{i-1}\left(\dot{x}_{i-1}-\dot{x}_{i}\right)-k_{i}\left(x_{i}-\right.\right. \\
\left.\left.x_{i+1}\right)-c_{i}\left(\dot{x}_{i}-\dot{x}_{i+1}\right)\right]+T_{i} / r=0 \quad i=1, n / 2+1
\end{gathered}
$$

对图 3 中各个单元的拓扑结构进行组集, 得到 如下所示输送机链传动系统的动力学模型

$$
\boldsymbol{M} \ddot{\boldsymbol{X}}+\boldsymbol{C} \dot{\boldsymbol{X}}+\boldsymbol{K X}=\boldsymbol{F}
$$

式中, $M$ 为质量矩阵; $\boldsymbol{F}$ 为力矢量; $\ddot{X}$ 为加速度矢 量; $\dot{X}$ 为速度矢量; $\boldsymbol{X}$ 为位移矢量; $\boldsymbol{K}$ 为刚度矩阵; $C$ 为阻尼矩阵。

\section{2 整机机电耦合模型}

3.2.1 整机动力学模型的建立

工作面输送机各部件动力学模型已经建立, 由 此可建立整机系统动力学模型 


$$
\left\{\begin{array}{l}
T_{\mathrm{M}}=\frac{2\left(\frac{T_{\mathrm{Mm}}-T_{\mathrm{Mm} 1} s_{\mathrm{m}}}{1-s_{\mathrm{m}}}+\frac{T_{\mathrm{Mm} 1}-T_{\mathrm{Mm}}}{1-s_{\mathrm{m}}} \cdot s\right)}{s /\left[S_{\mathrm{m}}\left(\frac{1-s_{\mathrm{m} 1}}{1-s_{\mathrm{m}}}+\frac{s_{\mathrm{m} 1}-s_{\mathrm{m}}}{1-s_{\mathrm{m}}} \cdot \frac{s}{s_{\mathrm{m}}}\right)\right]+s_{\mathrm{m}}\left(\frac{1-s_{\mathrm{m} 1}}{1-s_{\mathrm{m}}}+\frac{s_{\mathrm{m} 1}-s_{\mathrm{m}}}{1-s_{\mathrm{m}}} \cdot \frac{s}{s_{\mathrm{m}}}\right) / s} \\
s=1-\frac{n_{\mathrm{e}} p}{60 f} \\
T_{\mathrm{M}}-T_{\text {load }}=\frac{\pi I_{\mathrm{M}}}{30} \cdot \frac{\mathrm{d} n_{\mathrm{e}}}{\mathrm{d} t} \\
T_{\mathrm{M}} \cdot n_{\mathrm{e}}=T_{\mathrm{j}} \cdot n_{\mathrm{j}} \\
n_{\mathrm{j}}=n_{\mathrm{e}} / \eta \\
T_{1}=T_{\mathrm{j}} \\
T_{1}=I_{1} \ddot{\theta}_{1}-\left(F_{1}-F_{n}\right) r
\end{array}\right.
$$

式中, $T_{\mathrm{j}}$ 为减速器输出转矩; $n_{\mathrm{j}}$ 为减速器输出转速; $\eta$ 为减速器传动比。

变频电动机输出转矩 $T_{\mathrm{M}}$ 经过减速器作用在链 传动系统, 忽略减速器功率损失, 减速器输出即为 链轮的输入, 链轮旋转带动链条运动。启动后链条 张力 $F$ 作为输出负载反馈给电动机。

\section{2 .2 整机仿真模型的建立}

变频电动机的仿真模型可依据其动力学方程采用 $\mathrm{S}$ 函数建立。对减速器简化处理, 将其转动惯量折算到 链轮轴上。针对链传动系统, 依据状态方程建立其仿 真模型, 选取状态变量 $V_{1}=x_{1}, V_{2}=\dot{x}_{1}, V_{3}=x_{2}, y_{2}=V_{2}, V_{4}=$ $\dot{x}_{2}, \cdots, V_{2 n-1}=x_{n}, V_{2 n}=\dot{x}_{n}$; 输出变量为 $y_{1}=V_{1}, \cdots$, $y_{2 n-1}=V_{2 n-1}, y_{2 n}=V_{2 n}$ 。链传动系统状态方程为

$$
\left\{\begin{array}{l}
\dot{V}=A V+B U \\
y=C V
\end{array}\right.
$$

式中, $\boldsymbol{V}$ 为状态矢量; $\boldsymbol{U}$ 为控制矢量; $\boldsymbol{y}$ 为输出矢 量; $\boldsymbol{A}$ 为系数矩阵; $\boldsymbol{B}$ 为输入矩阵; $\boldsymbol{C}$ 为输出矩阵。 各自展开式可参考文献[7]。

合理选择接口变量, 再根据状态方程中各变量 间的关系连接各子系统得到整机机电耦合仿真模 型, 见图 4。

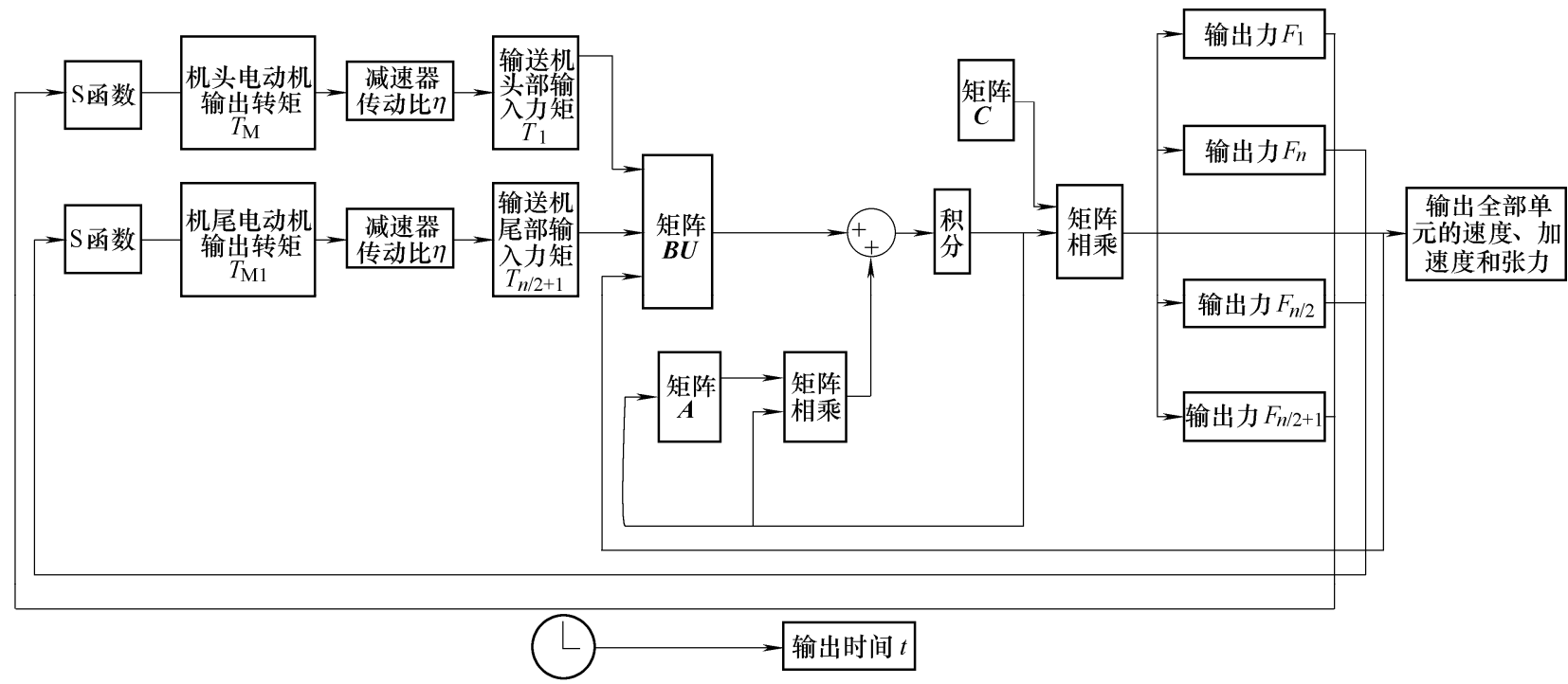

图 4 输送机整机机电耦合仿真模型

4 变频控制策略与性能评价指标

\section{1 变频控制策略}

变频电动机的控制模式可分为时序控制和反馈
控制。时序控制为开环控制, 随时间变化逐步将控 制信号作用于电动机; 反馈控制需利用电流、链段 动态张力等信息作为反馈信号, 是一种闭环控制。 目前工程上常用的是时序控制。变频器时序控制包 括变频启动时间和加速曲线模式的选取。 
变频启动时间与生产机械的惯性及工况有关, 在满足生产要求的条件下, 启动时间越短越好。

目前采用的加速曲线如图 5 所示, 主要有正 弦型、直线型、反 $\mathrm{S}$ 型和抛物线型。它们的启动 公式如下。

(1) 正弦型

$$
f= \begin{cases}f_{0} \sin \left(\frac{\pi}{2 T} t\right) & t<T \\ f_{0} & t \geqslant T\end{cases}
$$

(2) 直线型

$$
f= \begin{cases}f_{0} \frac{t}{T} & t<T \\ f_{0} & t \geqslant T\end{cases}
$$

(3) 反 $\mathrm{S}$ 型

$$
f=\left\{\begin{array}{lr}
f_{0} \frac{t}{T}-\frac{f_{0}}{2 \pi} \sin \left(\frac{2 \pi}{T} t\right) & t<T \\
f_{0} & t \geqslant T
\end{array}\right.
$$

（4）抛物线型

$$
f=\left\{\begin{array}{lr}
f_{0} \frac{t^{2}}{T^{2}} & t<T \\
f_{0} & t \geqslant T
\end{array}\right.
$$

式中, $f_{0}$ 为额定频率; $T$ 为变频启动时间。

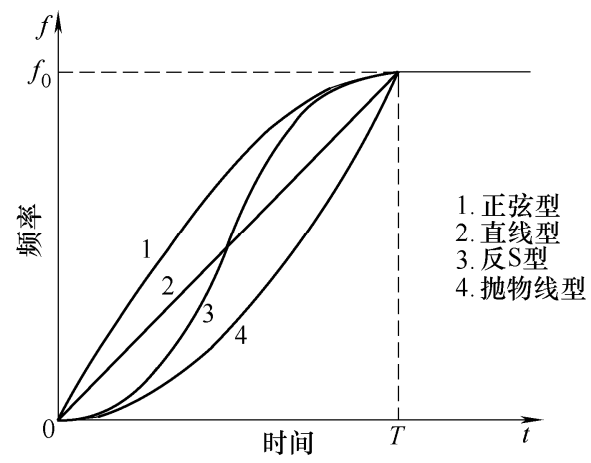

图 5 典型加速曲线

\section{2 输送机性能评价指标}

为评价输送机变频启动性能优劣, 采用如下指 标作为评价标准。

\subsection{1 峰值张力与最小张力}

峰值张力表征链段所受动态张力的最大值, 最 小张力指链条张力最小点处的张力值, 见式(16)。 峰值张力过大, 容易导致链条被拉断或链条的寿命 降低; 峰值张力越小, 链条磨损少, 延长链条寿命。 同时, 为防止链条松弛或堆链现象, 链条最小张力 应大于零, 从而实现链条张力和负荷的最佳匹配, 保护链条的安全。

$$
\left\{\begin{array}{c}
F_{\max }=\max \left(F_{1}, \cdots, F_{i}, \cdots, F_{n}\right) \\
F_{\min }=\min \left(F_{1}, \cdots, F_{i}, \cdots, F_{n}\right) \\
i=1,2, \cdots, n
\end{array}\right.
$$

式中, $F_{i}$ 为链段单元 $i$ 张力值。

\subsection{2 头尾电动机功率比}

头尾电动机功率比是指仿真过程中, 头部和尾 部电动机瞬时功率的比, 用式(17)表示。该比值随 时间的变化而变化, 并最终趋近于 1 , 可用一条曲 线来表示。该曲线反映了双驱动输送机头尾部电动 机的平衡性, 曲线偏离 1 越大, 说明头尾部电动机 功率越不平衡, 曲线稳定在 1 附近所需时间越长, 说明头尾部电动机功率平衡所需时间越长。头尾电 动机功率不平衡会造成头部和尾部电动机的寿命不 一，应尽量避免。

$$
i_{\mathrm{M}}=\frac{P_{\mathrm{t}}}{P_{\mathrm{w}}}
$$

式中, $i_{\mathrm{M}}$ 为头尾电动机功率比; $P_{\mathrm{t}}$ 为头部电动机的 功率; $P_{\mathrm{w}}$ 为尾部电动机的功率。

\subsection{3 电动机转矩标准差和振荡次数}

工作面输送机需要电动机在启动过程中平稳地 输出恒定转矩, 采用标准差和振荡次数来评定电动 机转矩的波动程度和稳定性。标准差反映转矩在竖 直方向的波动情况, 标准差越大, 波动越激烈; 标 准差越小, 波动越平稳。在一定时间内, 转矩穿越 其稳态值次数的一半定义为振荡次数, 其反映系统 时间响应的平稳性，振荡次数越少，波形越稳定。 转矩标准差和振荡次数可表示为

$$
\left\{\begin{array}{l}
S_{\mathrm{T}}=\sqrt{\sum_{j=1}^{m}\left(T_{j}-\bar{T}\right)^{2} / m} \\
N_{\mathrm{T}}=\frac{t_{s}}{2 \pi / \omega_{\mathrm{d}}}
\end{array}\right.
$$

式中, $S_{\mathrm{T}}$ 为转矩标准差; $T_{j}$ 为转矩在第 $j$ 个采样点 的值; $m$ 为采样点个数; $\bar{T}$ 为转矩平均值; $N_{\mathrm{T}}$ 为转 矩振荡次数; $t_{s}$ 为调整时间; $\omega_{\mathrm{d}}$ 为有阻尼振荡频率。

\subsection{4 链条张力标准差和振荡次数}

为保证输送机平稳启动, 链条张力在启动过程 中应波动平稳。若波动剧烈, 则引起系统加速度突 变, 导致附加动载荷。为此, 引入链条张力标准差 和振荡次数用来评价张力的波动情况。

$$
\left\{\begin{array}{l}
S_{\mathrm{F}}=\sqrt{\sum_{j=1}^{m}\left(F_{j}-\bar{F}\right)^{2} / m} \\
N_{\mathrm{F}}=\frac{t_{s}}{2 \pi / \omega_{\mathrm{d}}}
\end{array}\right.
$$


式中, $S_{\mathrm{F}}$ 为张力标准差; $F_{j}$ 为张力在第 $j$ 个采样点 的值; $\bar{F}$ 为张力平均值; $N_{\mathrm{F}}$ 为张力振荡次数。

\section{5 算例研究}

本文仿真采用 SGZ1000/2×1000 型输送机, 变 频驱动频率范围为 $0 \sim 50 \mathrm{~Hz}$, 链段划分为 60 单元, 具体参数见表 1 。

\section{表 1 SGZ1000/2×1000 型输送机组件参数}

\begin{tabular}{lc||lc}
\hline $\begin{array}{c}\text { YBSS-1000G 型三相 } \\
\text { 异步电动机参数 }\end{array}$ & 数值 & \multicolumn{1}{|c}{$\begin{array}{c}\text { 链传动 } \\
\text { 系统参数 }\end{array}$} & 数值 \\
\hline 电动机功率 $/ \mathrm{kW}$ & 1000 & 单位长度煤质量 $/(\mathrm{kg} / \mathrm{m})$ & 461.5 \\
额定转速 $/(\mathrm{r} / \mathrm{min})$ & 1489 & 输送长度 $/ \mathrm{m}$ & 299 \\
额定转矩 $/(\mathrm{N} \cdot \mathrm{m})$ & 6405 & 头尾链轮距离 $/ \mathrm{m}$ & 280 \\
线电压 $/ \mathrm{V}$ & 3300 & 链条长度 $/ \mathrm{m}$ & 602 \\
启动转矩/额定转矩 & 1.7 & 链环型号 & $42 \times 152$ \\
最大转矩/额定转矩 & 2.5 & 单位长度链条质量 $/(\mathrm{kg} / \mathrm{m})$ & 172.9 \\
转子电阻 $/ \Omega$ & 0.0638 & 铺设倾角 $/\left({ }^{\circ}\right)$ & $-5 \sim 5$ \\
\hline
\end{tabular}

\section{1 确定控制参数}

为得到最优控制策略, 需要对以下控制参数进 行合理选取。

(1) 预紧力。工作面输送机在运行过程中链条 只能承受拉力, 不能承受压力。为了输送机在稳定 工作状态下链条不出现松弛或发生堆链, 在输送机 正式运行前, 要选择合适的预紧力, 对链条进行预 紧。选取启动过程中机头、机尾电动机动态特性相 近且最小链段张力大于零的预紧力为最佳预紧力。

(2) 头尾延迟时间。双机驱动的输送机, 机尾 电动机先启动可以分担机头电动机的负担, 使头尾 电动机的负载更加均衡, 可以有效延长电动机的寿 命。选取可以使输送机头尾电动机功率平衡的时间 为最佳延迟时间。

(3) 加速曲线模式及变频启动时间。合理选取 加速曲线模式及变频启动时间, 能够有效地降低输 送机在启动过程中较大的加速度及加速度突变, 从 而避免产生较大动载荷, 减少对输送机的冲击。选 取能够使电动机转矩及链条张力波动平稳及链条峰 值张力较小的加速曲线及变频时间。

由以上分析可知, 预紧力与头尾延迟时间均影 响机头、机尾电动机负载平衡, 其是相互耦合的; 但其对加速曲线模式及变频启动时间几乎无影响。

\subsection{1 最优预紧力}

预紧力的大小与链条稳定运行时关键链段的张 力有关, 双机驱动的输送机关键链段单元张力分布 趋势如图 6 所示。其中 $F_{\mathrm{A}}$ 为链段第 1 单元所受张力, $F_{\mathrm{B}}$ 为链段第 $n / 2$ 单元所受张力, $F_{\mathrm{C}}$ 为链段第 $n / 2+1$
单元所受张力, $F_{\mathrm{D}}$ 为链段第 $n$ 单元所受张力。

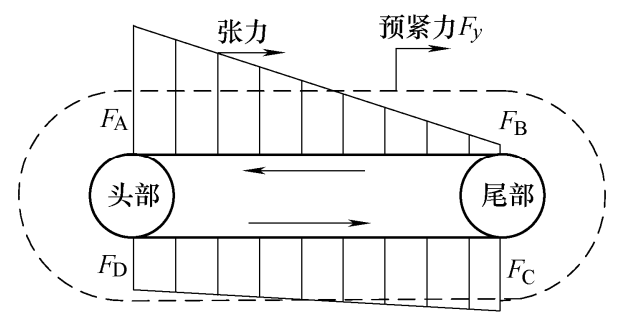

图 6 关键链段张力分布

为确定输送机在满载下合适的预紧力, 选取最 简单的直线型加速曲线, 变频时间 $100 \mathrm{~s}$, 进行无预 紧力仿真，仿真时间为 $150 \mathrm{~s}$ 。仿真得出无预紧力时 关键链段张力依次为: $F_{\mathrm{A}}=672 \mathrm{kN}, F_{\mathrm{B}}=0, F_{\mathrm{C}}=350$ $\mathrm{kN}, F_{\mathrm{D}}=220 \mathrm{kN}$ 。输送机预紧力初值估算公式为 $F_{y}=\left(F_{\mathrm{A}}+F_{\mathrm{B}}+F_{\mathrm{C}}+F_{\mathrm{D}}\right) / 4^{[18]}$ 。由此可知预紧力大 约在 $310 \mathrm{kN}$ 左右。再分别对预紧力为 $310 \mathrm{kN}, 320$ $\mathrm{kN}$ 进行仿真, 结果见图 7、8。

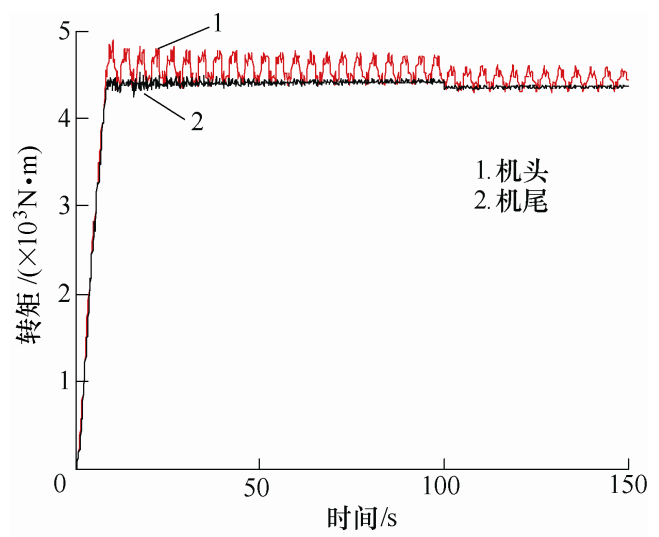

(a)头尾部电动机转矩

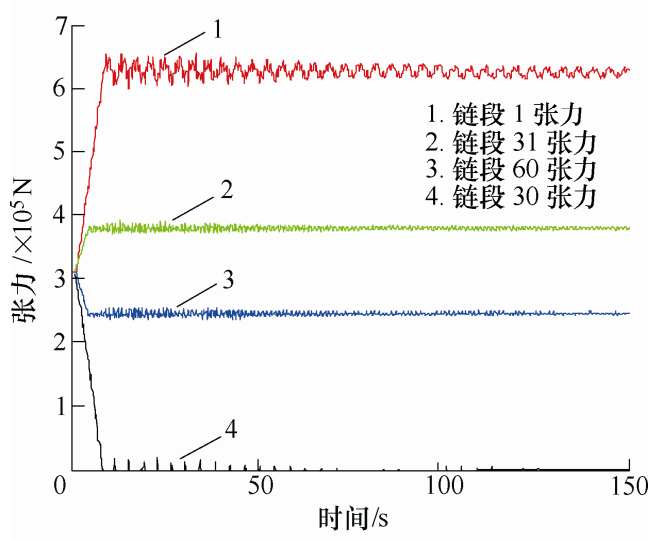

(b) 关键链段张力

图 7 预紧力为 $310 \mathrm{kN}$ 仿真结果

由图 7、8 仿真结果可以得出, 预紧力为 $310 \mathrm{kN}$ 时张力最小点 (关键链段 30)张力值在 0 左右振动, 头尾电动机功率比约为 1.08 ; 预紧力为 $320 \mathrm{kN}$ 时张 力最小点 (关键链段 30)张力值约为 $15 \mathrm{kN}$, 头尾电 动机功率比约为 1.01 。相比于预紧力为 $310 \mathrm{kN}$, 预 
紧力为 $320 \mathrm{kN}$ 时最小张力大于零, 可以避免链条堆 链, 且头尾电动机功率比接近 1 , 头尾部电动机负 载分配更均衡。

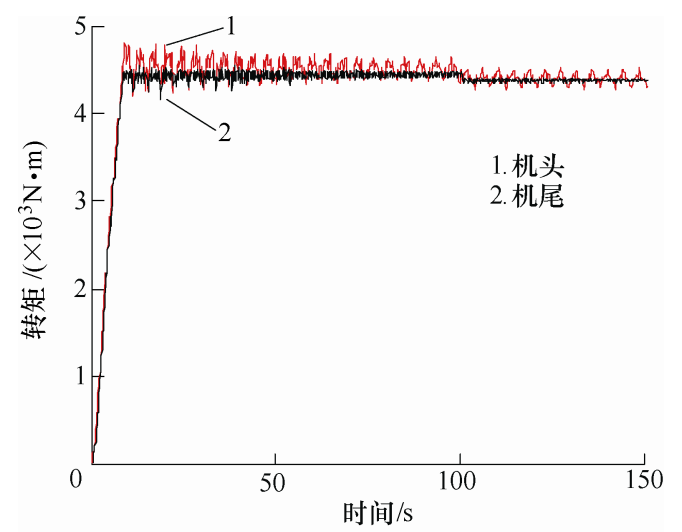

(a)头尾部电动机转矩

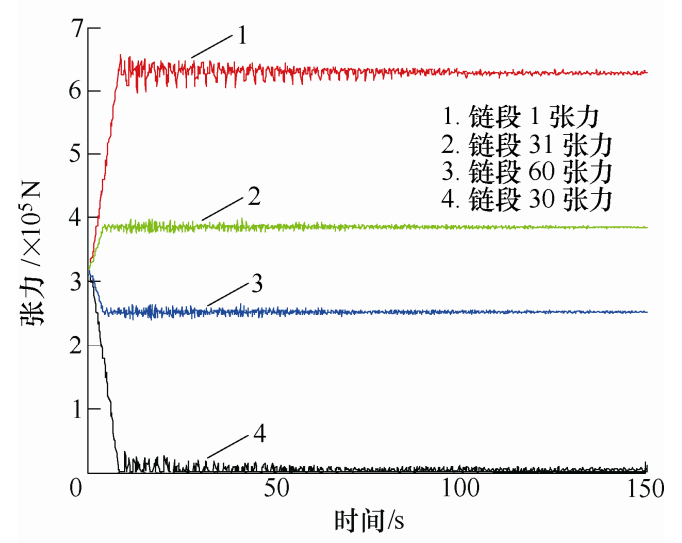

(b) 关键链段张力

图 8 预紧力为 $320 \mathrm{kN}$ 仿真结果

综上, 随着预紧力的增加, 头尾部电动机负载 更均衡, 但链条各点张力随之增加, 过大的预紧力 可能导致链条被拉断。因此, 在保证张力最小点张 力值不为零的情况下, 预紧力越小越好。在上述仿 真条件下, 应选取预紧力为 $320 \mathrm{kN}$, 不应再大。

\subsection{2 头尾延迟时间}

由于预紧力与头尾延迟时间相互耦合, 即头尾 延迟时间与预紧力施加大小有关。为了确定特定预 紧力下合适的头尾延迟时间, 进行如下仿真。分别 在预紧力 $310 \mathrm{kN}$ 情况下进行同时启动、机头晚启动 $1.5 \mathrm{~s}$ 、机头晚启动 $3 \mathrm{~s}$ 的仿真, 在预紧力为 $320 \mathrm{kN}$ 条件下进行同时启动、机头晚启动 $1 \mathrm{~s}$ 、机头晚启动 $2 \mathrm{~s}$ 的仿真, 其余仿真条件同上节。仿真结果如图 9、 10 所示。

由仿真结果可以看出, 预紧力为 $310 \mathrm{kN}$ 时, 机 头晚启动 $1.5 \mathrm{~s}$ 时功率比接近 1 , 头尾电动机功率基 本达到平衡; 预紧力为 $320 \mathrm{kN}$ 时, 机头晚启动 $1 \mathrm{~s}$ 时头尾电动机功率已基本平衡, 继续增加晚启动时 间, 反而使得尾部电动机负载增大, 起到适得其反 的作用。

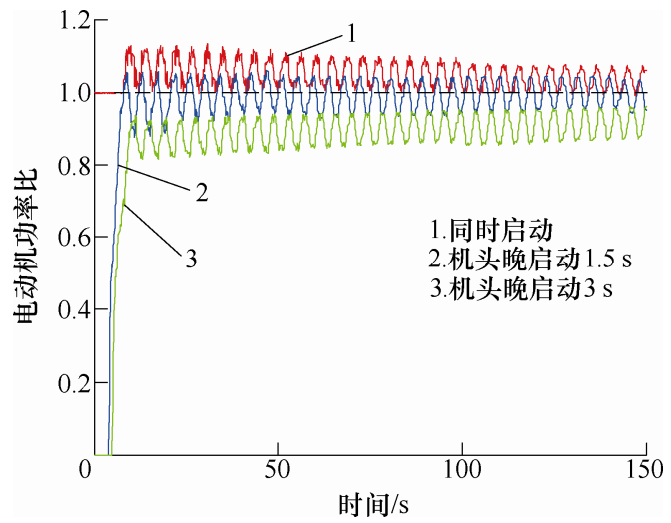

图 9 预紧力为 $310 \mathrm{kN}$ 时头尾不同延迟时间仿真结果

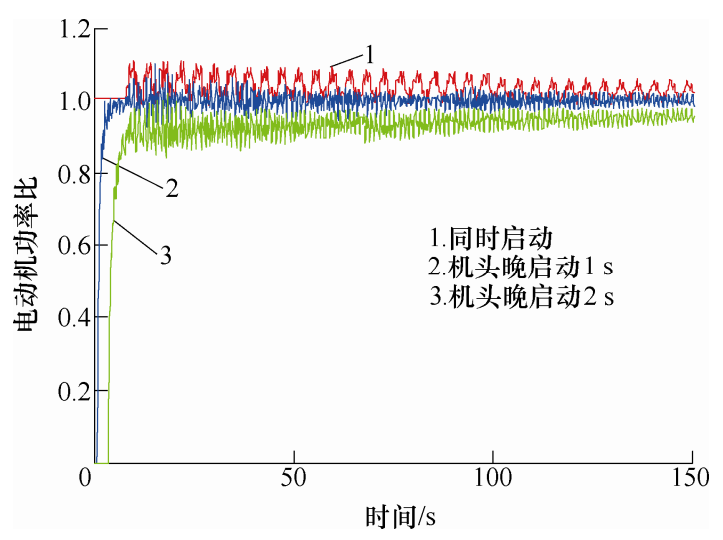

图 10 预紧力为 $320 \mathrm{kN}$ 时头尾不同延迟时间仿真结果

可见, 预紧力越大, 头部电动机最佳晚启动时 间越短。且如果延迟时间太短, 机头的输出功率大 于机尾的输出功率; 如果延迟时间过长, 机尾的输 出功率大于机头的, 处于单机拖动状态。

综上, 应选择与预紧力搭配合适的头尾延迟时 间, 从而使系统既不发生堆链, 又能使头尾平均分 摊负载。即在 $320 \mathrm{kN}$ 预紧力作用下, 选择晚启动时 间 $1 \mathrm{~s}$ 最合适。

\section{1 .3 加速曲线模式}

施加最优预紧力, 头尾延迟时间 $1 \mathrm{~s}$, 针对不同 加速曲线进行满载工况仿真, 变频启动时间均设为 $100 \mathrm{~s}$, 总仿真时间 $150 \mathrm{~s}$, 仿真结果如图 11、表 2 所示。

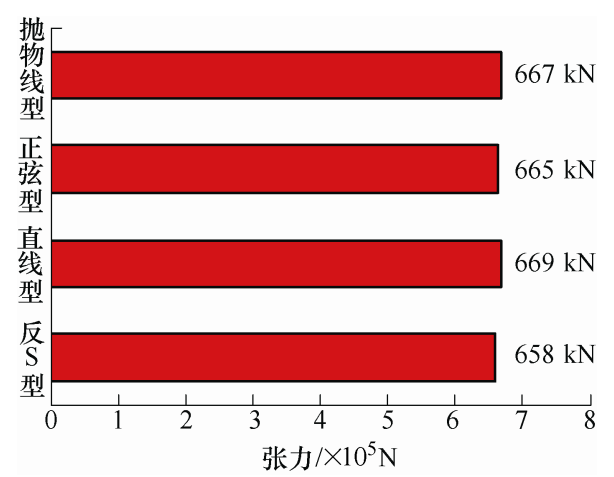

图 11 不同加速曲线链条峰值张力 
表 2 不同加速曲线转矩及张力的标准差与振荡次数

\begin{tabular}{ccccc}
\hline 加速曲线 & $\begin{array}{c}\text { 转矩标准 } \\
\text { 差 } S_{T} /(\mathrm{N} \cdot \mathrm{m})\end{array}$ & $\begin{array}{c}\text { 转矩振荡 } \\
\text { 次数 } N_{T}\end{array}$ & $\begin{array}{c}\text { 张力标准 } \\
\text { 差 } S_{F} / \mathrm{N}\end{array}$ & $\begin{array}{c}\text { 张力振荡 } \\
\text { 次数 } N_{F}\end{array}$ \\
\hline 反 $\mathrm{S}$ 型 & 91 & 63 & 4809 & 122 \\
直线型 & 93 & 99 & 6281 & 153 \\
正弦型 & 155 & 84 & 10152 & 132 \\
抛物线型 & 87 & 77 & 4875 & 129 \\
\hline
\end{tabular}

注: 双机驱动输送机时链段 1 处承载最大, 选取此单元作为评价 标准。

由图 11 看出四种加速曲线中反 $\mathrm{S}$ 型的峰值张力 最小, 即系统加速度最大值最小; 分析表 1 可得, 相比于其他三种加速曲线, 反 $\mathrm{S}$ 型振荡次数最少, 标准差较小, 表明其电动机转矩和链条张力波动小, 稳定性好。因此, 反 $\mathrm{S}$ 型加速曲线最合适。

\subsection{4 变频启动时间}

施加最优预紧力, 头尾延迟时间 $1 \mathrm{~s}$, 在反 $\mathrm{S}$ 型 加速曲线下满载启动, 分别设置启动时间为 $50 \mathrm{~s}$ 、 $100 \mathrm{~s} 、 150 \mathrm{~s}$, 总仿真时间 $150 \mathrm{~s}$, 仿真结果如图 12 、 表 3 所示。

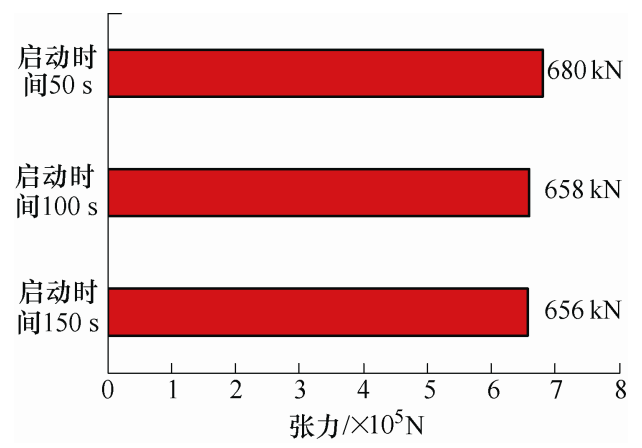

图 12 不同启动时间链条峰值张力

表 3 不同启动时间转矩及张力的标准差与振荡次数

\begin{tabular}{ccccc}
\hline 启动时间 $/ \mathrm{s}$ & $\begin{array}{c}\text { 转矩标准差 } \\
S_{T} /(\mathrm{N} \cdot \mathrm{m})\end{array}$ & $\begin{array}{c}\text { 转矩振荡 } \\
\text { 次数 } N_{T}\end{array}$ & $\begin{array}{c}S_{F} / \mathrm{N} \\
S_{\text {na }} \text { 准差 }\end{array}$ & $\begin{array}{c}\text { 张力振荡 } \\
\text { 次数 } N_{F}\end{array}$ \\
\hline 50 & 206 & 118 & 12223 & 150 \\
100 & 91 & 63 & 4806 & 122 \\
150 & 82 & 90 & 5471 & 115 \\
\hline
\end{tabular}

由图 12 可以得出, 链条峰值张力随着启动时间 的增加逐渐减小; 分析表 2 发现, 启动时间 $50 \mathrm{~s}$ 时 转矩和张力波动剧烈, 稳定性差; 启动时间 $100 \mathrm{~s}$ 与 $150 \mathrm{~s}$ 时波动较小, 稳定性较高。但启动时间过 长, 则启动缓慢, 造成浪费。综上, 选取启动时间 为 $100 \mathrm{~s}$ 最合理。

\section{2 与偶合器对比}

通过以上分析可以得到变频驱动的最优控制策 略, 即最优预紧力 $320 \mathrm{kN}$, 头尾延迟时间 $1 \mathrm{~s}$, 加 速曲线反 $\mathrm{S}$ 型，变频启动时间 $100 \mathrm{~s}$ 。

在最优控制策略下, 将变频驱动仿真得到的结 果与同工况下液力偶合器驱动的仿真结果进行对
比, 总的仿真时间为 $150 \mathrm{~s}$, 结果如图 13、14 所示。

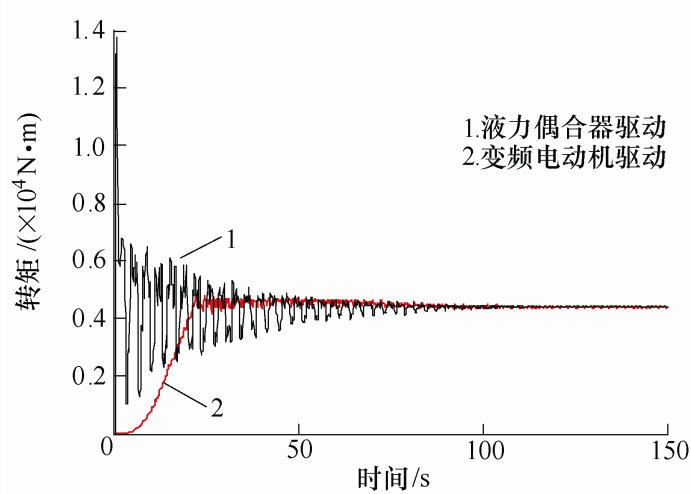

(a) 机头电动机输出转矩

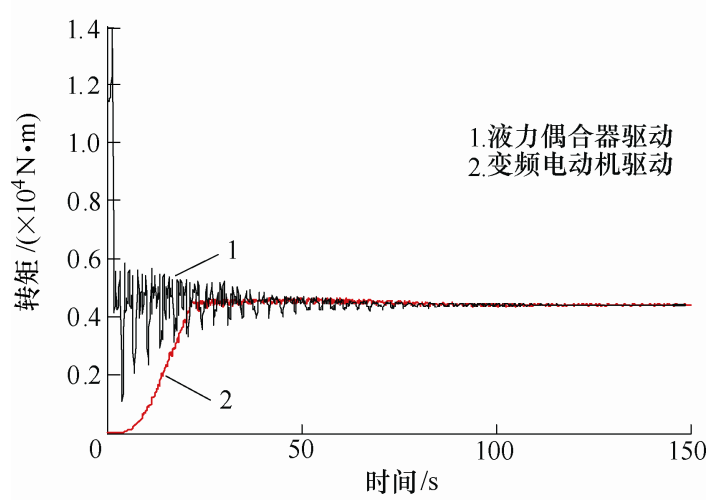

(b) 机尾电动机输出转矩

图 13 两种驱动方式机头机尾电动机输出转矩

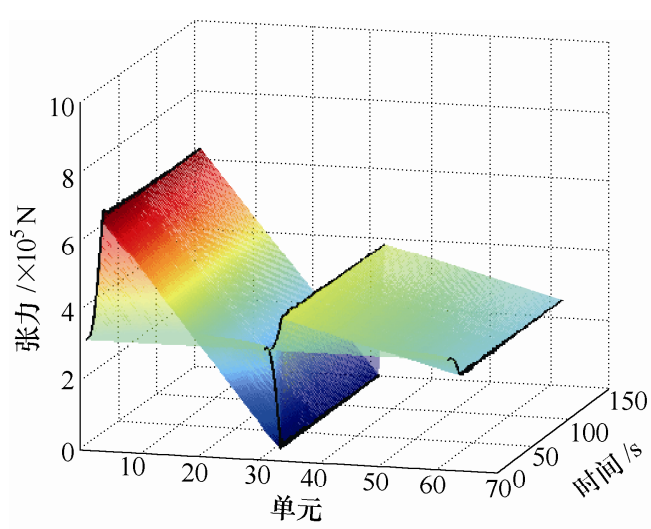

(a) 变频电动机驱动各链段张力

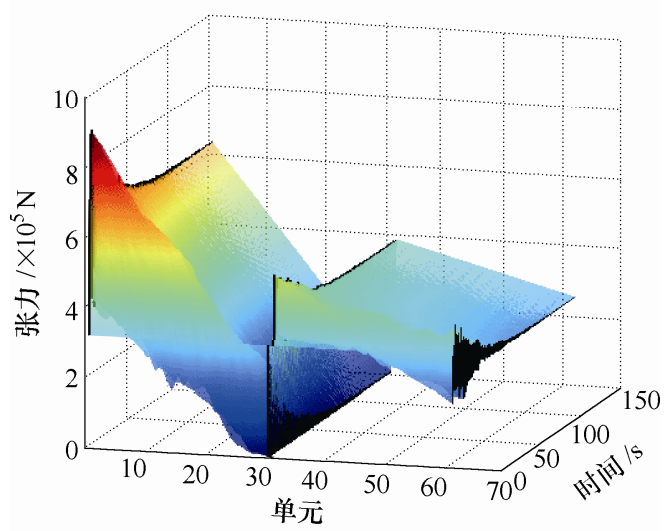

(b) 液力偶合器驱动各链段张力

图 14 两种驱动方式全部链段单元张力 
由图 13 可得, 液力偶合器驱动时机头、机尾电 动机转矩在 $0 \sim 3 \mathrm{~s}$ 时突变明显, 且波动剧烈。这是 由于液力偶合器驱动是电动机先启动, 达到额定转 速后再与负载相连; 变频驱动时电动机转矩从零开 始上升到额定值, 说明其为带载启动, 且转矩波动 明显较小。电动机近似为恒转矩输出, 值为 4424 $\mathrm{N} \cdot \mathrm{m}$, 恒转矩输出有利于解决重载难启动。且液力 偶合器驱动时机头、机尾电动机在启动前 $75 \mathrm{~s}$ 时负 载不均衡, 转矩波动剧烈; 而变频驱动在启动过程 中能很好地保持机头、机尾电动机负载均衡, 两电 动机能够同时发挥最大的特性。

由图 14 可知, 由于施加了预紧力, 使得两种驱 动方式链条最小张力均大于 0 , 可避免产生堆链。 变频驱动链条最大张力为 $658 \mathrm{kN}$, 相比于液力偶合 器驱动最大张力 $898 \mathrm{kN}$, 减小了约 $27 \%$, 从而大大 减少了链条磨损, 延长链条寿命。链条关键点张力 用图中黑线表示, 可看出液力偶合器驱动时链条张 力波动剧烈, 变频驱动时链条张力在启动过程中波 形平稳, 因此变频驱动在启动过程中产生的动载荷 较小, 能够明显减小对输送机的机械冲击。

\section{6 结论}

（1）依据机械特性建立了变频电动机模型, 基 于有限段法建立了链传动系统动力学模型, 从而建 立了输送机整机机电耦合模型。

(2) 为评价输送机变频启动性能优劣, 给出了峰 值张力、最小张力、标准差及振荡次数等评价指标。

(3) 通过对满载启动工况进行仿真, 确定了最 优预紧力、头尾延迟时间、加速曲线模式及变频启 动时间等参数, 从而得到了变频驱动最优控制策略。

(4) 为工作面输送机软启动方式的选取及变频 电动机控制策略的研究提供参考依据。

\section{参 考 文 献}

[1] DU P G E, LIEBENBERG L, MATHEWS E H. The use of variable speed drives for cost-effective energy savings[J]. Applied Energy, 2013， 111: 16-27.

[2] JUNGHANS D. Modern drive technology for belt conveyors[J]. Bulk Solids Handling, 2001, 21(4): 438-441.

[3] WAUGE D H. Modeling of an armoured face conveyor[D]. Queensland: The University of Queensland, 2002.

[4] DOLIPSKI M, REMIORZ E, SOBOTA P. Dynamics of non-uniformity loads of AFC drives[J]. Arch. Min. Sci,
2014(59): 155-168

[5] 宋伟刚, 王丹. $7.6 \mathrm{~km}$ 长距离带式输送机系统动力学的 计算机仿真[J]. 煤炭学报, 2004(2): 249-253.

SONG Weigang, WANG Dan. The dynamic computer simulation of long distance belt conveyor with $7.6 \mathrm{~km}[\mathrm{~J}]$. Journal of China Coal Society, 2004(2): 249-253.

[6] 宋伟刚, 柳洪义, 王鹰. 带式输送机动力学及其计算机 仿真的研究 [J]. 机械工程学报, 2003, 39(9): 133-138. SONG Weigang, LIU Hongyi, WANG Ying. Research on dynamic and computer simulation of the belt conveyor[J]. Chinese Journal of Mechanical Engineering, 2003, 39(9): 133-138.

[7] NIE R, HE B Y, ZHANG L H. Modeling of the transmission system in conveying equipment based on Euler method with application[J]. Proceedings of the Institution of Mechanical Engineers, Part K: Journal of Multi-body Dynamics, 2014, 228: 294-306.

[8] NIE R, HE B Y, YUAN P F, et al. Novel approach to and implementation of design and analysis of armored face conveyor power train[J]. Science China Technological Sciences, 2015, 58: 1-16.

[9] 车林仙. 多样性保持离散差分进化算法及齿轮传动优 化应用 [J]. 机械工程学报，2016，52(21): 44-55.

CHE Linxian. Discrete differential evolution with diversity maintenance strategies and its application in optimization design for gear transmission[J]. Chinese Journal of Mechanical Engineering, 2016, 52(21): 44-55.

[10] CHEN Y B, GUO H T, FU Z M, et al. Design and analysis of the gemini chain system in dual clutch transmission of automobile[J]. Chinese Journal of Mechanical Engineering, 2015，28(1): 38-45.

[11] 孙阳辉. 工作面输送机传动特性与参数匹配研究 [D]. 天津: 天津大学, 2012.

SUN Yanghui. Research on transmission characteristics and parameter matching of an face conveyor[D]. Tianjin: Tianjin University, 2012.

[12] 毛君, 师建国, 张东升, 等. 重型刮板输送机动力建模 与仿真[J]. 煤炭学报, 2008, 33(1): 103-106.

MAO Jun, SHI Jianguo, ZHANG Dongsheng, et al. Dynamic modeling and simulation of heavy scraper conveyor[J]. Journal of China Coal Society, 2008, 33(1): 103-106.

[13] 张东升, 毛君, 刘占胜. 刮板输送机启动及制动动力学 特性仿真与实验研究 [J]. 煤炭学报, 2016(2): 513-521. ZHANG Dongsheng, MAO Jun, LIU Zhansheng. Dynamics simulation and experiment on the starting and braking of scraper conveyor[J]. Journal of China Coal Society, 2016(2): 513-521.

[14] 李小凡. 刮板输送机变频驱动控制系统的研究 [D]. 太 
原: 太原理工大学, 2013.

LI Xiaofan. The scraper conveyor variable frequency drive control system[D]. Taiyuan: Taiyuan University of Technology, 2013.

[15］樊启高. 综采工作面 “三机” 控制中设备定位及任务协 调研究[EB/OL]. [2017-05-19]. https: //wenku.baidu.com/ view/33d917ae783e0912a3162abf.html.

FAN Qigao. Research on equipment positioning and task coordination in "three machines" control of fully mechanized coal face[EB/OL]. [2017-05-19]. https: // wenku.baidu.com/view/33d917ae783e0912a3162abf.html.

[16] 谢丽蓉, 王智勇, 竹勤. 鼠笼异步电动机机械特性的研 究[J]. 中国电动机工程学报, 2008(21): 68-72.

XIE Lirong, WANG Zhiyong, CHAO Qin. Research on the mechanical characteristic of squierrelcage asynchronous motors $[\mathrm{J}]$. Proceedings of the CSEE, 2008(21): 68-72.

[17] 何柏岩, 孙阳辉, 聂锐, 等. 矿用刮板输送机圆环链传 动系统动力学行为研究 [J]. 机械工程学报, 2012 , 48(17): 50-56.
HE Baiyan, SUN Yanghui, NIE Rui, et al. Dynamic behavior analysis on the ring chain transmission system of an armoured face conveyor[J]. Journal of Mechanical Engineering, 2012, 48(17): 50-56.

[18] 张国柱. 综采输送机[M]. 北京: 煤炭工业出版社, 1986. ZHANG Guozhu. Fully mechanized conveyor[M]. Beijing: China Coal Industry Publishing House, 1986.

作者简介: 李阳, 男, 1991 年出生。主要研究方向为机电系统动力学建 模与仿真。

E-mail: liyang5537@tju.edu.cn

何柏岩(通信作者), 男, 1973 年出生, 博士, 教授, 博士研究生导师。 主要研究方向为机械多体动力学与控制。

E-mail: hebaiyan@tju.edu.cn

袁鹏飞, 男, 1988 年出生, 博士研究生。主要研究方向为系统动力学建 模与仿真, 结构可靠性。

E-mail: hbgdypf@163.com

聂锐, 女, 1990 年出生, 博士研究生。主要研究方向为机电系统动力 学建模与仿真, 参数优化。

E-mail: nierui_ok@126.com

李国平, 男, 1963 年出生, 博士, 研究员级高级工程师。主要研究方向 为刮板输送机设计与制造。

E-mail: lgp@zmm.com 